\title{
Fallen will gelernt sein
}

\section{Sturzprophylaxe in der Altenhilfe}

\author{
Bianca Schrader
}

Bianca Schrader ist Referentin für Bewegungsprogramme im Team Altenhilfe und Gesundheitsförderung im Generalsekretariat des Deutschen Roten Kreuzes in Berlin.

E-Mailschradeb@drk.de
Stürze alter Menschen in ibrer Wohnung sind oft der Beginn eines langen Leidenwegs, der nicht selten im Pflegeheim endet. Die finanziellen Kosten für Akutversorgung und Rehabilitation von Schenkelhalsfrakturen werden in Deutschland auf über zwei Milliarden Euro geschätzt. Das Deutsche Rote Kreuz bietet zur Sturzprophylaxe Beratungskurse an.

Ein Blick auf die veränderte Altersstruktur in Deutschland zeigt, dass unsere Gesellschaft im zunehmenden Maße mit der Aufgabe konfrontiert ist, eine im Durchschnitt immer älter werdende Bevölkerung, weiterhin aktiv am Leben teilhaben zu lassen und dem Einzelnen ein selbstständiges Leben zu ermöglichen. Prävention und Gesundheitsförderung können chronische Krankheiten und Multimorbidität in ihrem Verlauf positiv beeinflussen. Entsprechende Interventionen führen nachweislich zu einer Stärkung der Lebensqualität und Selbständigkeit im Alter.

Die Auseinandersetzung mit dem Thema Altern und deren Folgen führt in der aktuellen Diskussion zwangsläufig auch dazu, die damit im Zusammenhang stehende Erhöhung der Kosten im Gesundheitswesen zu betrachten. Dabei wird deutlich, dass unter anderem durch Stürze und deren Folgen, neben den hohen persönlichen Kosten für den betroffenen Menschen, das Gesundheitssystem stark belastet wird.

Für Akutversorgung und Rehabilitation entstehen pro Schenkelhalsfraktur circa 15.000 Euro Kosten (Runge \& Schacht, 1999). Bei 135.000 Schenkelhalsfrakturen jährlich in Deutschland (Statistisches Bundesamt 2002) bedeutet das eine Belastung des Gesundheitssystems von über zwei Milliarden Euro im Jahr.

Um dieser Entwicklung entgegenzuwirken - vor allem zur Stärkung persönlicher Ressourcen der älteren Mitmenschen -, tragen gezielte Interventionen der Sturz- prophylaxe bei. Dabei ist es besonders wichtig, lange vor der Pflegebedürftigkeit anzusetzen.

Das Deutsche Rote Kreuz (DRK) führt seit über 40 Jahren Bewegungsangebote als Teil der Sozialarbeit durch. Das DRK-Programm »Bewegung bis ins Alter « enthält neben der Gymnastik die Bewegungssäulen Wassergymnastik und Tanzen und wird deutschlandweit mit 8.000 vorwiegend ehrenamtlichen DRK-Übungsleitern durchgeführt. Das Deutsche Rote Kreuz erreicht wöchentlich 350.000 Menschen mit den Bewegungsprogrammen in Ortsvereinen, Kreisverbänden und in Senioreneinrichtungen.

Das Deutsche Rote Kreuz setzt sich über die Expertenstandards Sturzprophylaxe (vgl. Kasten, Seite 232) in der Pflege hinaus intensiv mit dem Thema »Stürze verhindern « und »Sturzfolgen minimieren « aktiv auseinander. Die Tatsache, dass sich mit dem Thema Sturz außerhalb der stationären oder häuslichen Pflege beschäftigt wird, zeigt deutlich den präventiven Gedanken auf. Durch Fortbildungen zu diesem Thema werden DRKÜbungsleiter Gymnastik befähigt, die Teilnehmer der Bewegungsgruppen zur Kräftigung der sturzrelevanten Muskulatur anzuleiten, Gleichgewicht und Beweglichkeit $\mathrm{zu}$ trainieren und ein bewegtes Konditionstraining durchzuführen. Durch eine »Fallschule« können die Teilnehmenden lernen, wie im Ernstfall Sturzfolgen minimiert werden können. Des weiteren enthält das DRK-Angebot Informationen zur Vorbeugung von Stolperstellen im Umfeld, Hinweise auf die Überprüfung der Hör- und Sehfähigkeit, der Medikamenteneinnahme und Hinweise zur Überprüfung des Schuhwerks. Diese intrinsischen und extrinsischen Faktoren haben einen spürbaren Einfluss auf das Sturzrisiko und können durch gezieltes Training und Behebung der Risikofaktoren erheblich Stürzen und Sturzgeschehen vorbeugen. 
Vorreiter für die DRK-Sturzprophylaxe ist der DRK-Landesverband Hessen, der in einem Modellprojekt seine Übungsleiter durch Dr. Nadja Schott geschult hat. Dank der hohen Akzeptanz der Teilnehmerinnen und Teilnehmer in Hessen konnte die Idee in die Breite getragen werden konnte.
In der Ottawa-Charta (1986) werden Handlungsbereiche und Strategien zur Umsetzung von Gesundheitsförderung nach dem ressourcenorientierten Ansatz beschrieben. Die persönlichen Kompetenzen des Einzelnen sollen gestärkt, gesundheitsbezogene Gemeinschaftsaktionen gefördert und Gesundheitsdienste neu orga-

rem Lebensraum erfasst und die Interventionen darauf angepasst. Die Durchführung der Sturzprophylaxegymnastik im DRK-Ortsverein in Verbindung mit ehrenamtlichem Engagement ist ein gelebtes Beispiel für Gesundheitsförderung als Intervention in soziale Systeme.

\section{Resümee}

\section{"Beratung ist besonders erfolgreich, wenn sie im Alltag des Menschen verankert ist"}

Die Ausbildung zum DRK-Übungsleiter erfolgt in 160 Stunden und ist damit einer Ausbildung beim Turnerbund oder Landessportbund gleichzusetzen. Die Übungsleiter können im Anschluss an ihre Ausbildung ein spezielles Fortbildungsangebot zur Sturzprophylaxe bei ihrem Landesverband besuchen, um gezielt Elemente der Sturzprophylaxe in die DRK-Gymnastik einfließen zu lassen.

Von 23 befragten Einrichtungen der DRK-Altenhilfe meldeten zur Fachtagung Altenhilfe des Deutschen Roten Kreuzes im April 2009 alle Einrichtungsvertreter einen erhöhten Bedarf an zielgruppenspezifischen Interventionen in diesem Themenfeld. Die Workshopteilnehmer führten bereits vereinzelt DRK-Sturzprophylaxe-Angebote mit DRK-Übungsleitern durch und gaben an, dieses Angebot zukünftig vermehrt bei Ihren Orts- und Kreisverbänden zukünftig mit DRKÜbungsleitern abzufordern. nisiert werden. Für die Durchführung dieser Aufgaben wird das Setting, der alltägliche Lebensraum eines Menschen, empfohlen. Es hat sich nämlich gezeigt, dass soziale Kontakte und die Integration in Gruppen oder Settings einen Einfluss darauf haben, wie erfolgreich beispielsweise Senioren ein selbstständiges Leben im eigenen Heim führen können. Präventions- und gesundheitsfördernde Angebote in einem Setting haben daher vermutlich nicht nur eine unmittelbare vorbeugende und schützende Funktion für die Gesundheit, sondern sie dienen auch dem Erhalt der Selbstständigkeit. Sie bieten ebenfalls eine Möglichkeit zur sozialen Teilhabe und tragen zu einer höheren Lebensqualität bei. Deshalb ist es besonders sinnvoll, Prävention und Gesundheitsförderungsmaßnahmen wie die Sturzprophylaxe in einem Setting zu konzipieren und durchzuführen. Es werden die individuellen Bedürfnisse der Menschen in ih-
Die Handlungsbereiche Unterstützung gesundheitsbezogener Gemeinschaftsaktivitäten und die Förderung der Entwikklung persönlicher Kompetenzen sowie die Schaffung gesundheitsförderlicher Lebenswelten wird im Deutschen Roten Kreuz mit den Angeboten der Bewegungsprogramme umgesetzt. Es werden auf der Verhaltensebene persönliche Kompetenzen bei den Teilnehmenden gefördert, die weit über die persönlichen Folgen hinausgehen und damit eine wirtschaftliche Entlastung zur Folge haben. Eine aktive Beteiligung und Einflussnahme der Bürgerinnen und Bürger hinsichtlich der Gestaltung der Gesundheitsbedingungen im häuslichen Umfeld werden forciert und die sozialen Netzwerke zur gegenseitigen Hilfe und Unterstützung ausgebaut.

Die DRK-Bewegungsprogramme mit dem Schwerpunkt Sturzprophylaxe tragen innenwirksam zur Vernetzung der ambulanten und stationären Altenhilfe und zur Wahrnehmung der Hilfeleistungskette bei und machen die Querschnittsaufgabe der Gesundförderung deutlich.

\section{Der Expertenstandard Sturzprophylaxe}

in der Pflege hat zum Ziel, Stürze und Sturzfolgen zu vermeiden, indem ursächliche Risiken und Gefahren erkannt und nach Möglichkeit minimiert werden. Er richtet sich an alle Pflegefachkräfte, die Patienten oder Bewohner entweder in der häuslichen Umgebung oder in einer Einrichtung der stationären Gesundheitsversorgung oder der Altenhilfe betreuen. Dieser nationale Expertenstandard soll das Bewusstsein auf die professionelle Sturzprophylaxe in der Pflege lenken. [...] Der Expertengruppe des Deutschen Netzwerks für Qualitätsentwicklung in der Pflege (DNQP), das den Expertenstandard herausgegeben hat, gehörten 14 Personen an, darunter auch ein Patientenvertreter. Insgesamt wurden 252 Literatur-Titel berücksichtigt und analysiert. Der Expertenstandard besteht aus 18 Struktur-, Prozess- und Ergebniskriterien und wurde im Februar 2006 veröffentlicht.

Quelle: http://www.pflegewiki.de/wiki/Expertenstandard_Sturzprophylaxe 
"Das Gefühl von Gesundheit erwirbt man sich nur durch Krankheit. " Georg Christoph Lichtenberg, deutscher Naturwissenschaftler und Schriftsteller (1742-1799)

"Die größte Behinderung des Lebens liegt darin, ständig auf die Gesundheit zu achten." Platon, griechischer Philosoph (427 v. Chr.-347 v. Chr.)

"Etwas Gesundheit ab und zu ist das beste Heilmittel."

Friedrich Nietzsche, deutscher Philosoph (1844-1900)

„Hollywood ist voller fünfzigjähriger Männer, die sich so gewissenhaft und hingebungsvoll pflegen, dass sie wie sehr gesunde Sechzigjährige aussehen." Peter Ustinov, britischer Schauspieler und Schriftsteller (geb. 1921)

"Gesund ist, wenn ich nur abends -

und nicht auch tagsüber - ins Bett muss."

Schülerin, 9 Jahre

"Jede Gesundheit ist gemeinlich tödlich, wenn sie lange anhält, etwa 70, 80 Jahre." Jean Paul, deutscher Schriftsteller (1763-1825)

"Wir sind bestrebt, die uns durch Gott geschenkte Gesundheit der Luft durch unsere Vorsorge, so weit uns dies möglich, rein zu erhalten. Wir verfügen deshalb, dass es niemandem gestattet ist, in Gewässern, die weniger als eine Meile von einer Ansiedlung entfernt liegen, Flachs oder Hanf zu wässern, weil dadurch die Beschaffenheit der Luft ungünstig verändert wird." Kaiser Friedrich II. im Jahre 1231 